\title{
Cerebral Venous Thrombosis: What's New?
}

\author{
Diana Aguiar de Sousa ${ }^{1,2,3}$ \\ 1 Department of Neurosciences and Mental Health (Neurology), \\ Hospital Santa Maria - CHULN, University of Lisbon, Lisbon, Portugal \\ 2 Faculdade de Medicina, Universidade de, Lisboa, Portugal \\ 3 Instituto de Medicina Molecular João Lobo Antunes, Lisbon, Portugal
}

\begin{abstract}
Address for correspondence Diana Aguiar de Sousa, Department of Neurosciences and Mental Health (Neurology), Hospital Santa Maria, University of Lisbon, Av. Prof. Egas Moniz, 1649-028-Lisbon, Portugal (e-mail: dianasousa@campus.ul.pt).
\end{abstract}

Hämostaseologie 2021;41:25-30.

$$
\begin{aligned}
& \text { Abstract } \\
& \text { Keywords } \\
& \text { - cerebral venous } \\
& \text { thrombosis } \\
& \text { - brain lesion } \\
& \text { - recanalization } \\
& \text { - risk factor } \\
& \text { - anticoagulation }
\end{aligned}
$$

Thrombosis of the cerebral veins and sinuses (CVT) is a distinct cerebrovascular disorder that, unlike arterial stroke, most often affects children and young adults, especially women. In this review, we will summarize recent advances on the knowledge of patients with CVT.

\section{Epidemiology}

Cerebral venous thrombosis (CVT) is a less common form of stroke. In the largest published prospective multinational cohort of patients with CVT-the International Study on Cerebral Vein and Dural Sinus Thrombosis (ISCVT), ${ }^{1}$ the proportion of females was $75 \%$ and the median age at the thrombotic event was in the fourth decade of life. Other cohorts, from all over the world, found similar trends, ${ }^{2}$ with most adults with CVT being aged 20 to 50 years and less than $10 \%$ older than 65 years. $^{3}$ Importantly, the female predominance is restricted to the fertile age, as it is not seen in pediatric cohorts or in elderly patients. ${ }^{4}$ The most accurate estimates concerning incidence of CVT come from highincome countries, where two studies conducted with this aim indicated an overall prevalence of 13 to 16 cases per million per year. ${ }^{5}$ Also, a recent retrospective cohort study from the United States reported an incidence between 14 and 20 cases per million population (20-27 cases per million in females). ${ }^{6}$ Of note, this study also described an increase in incidence over time in older females and males, and a higher incidence rate in black Americans.

\section{Clinical and Imaging Manifestations}

Knowledge on the presentation and prognosis of CVT was very much improved in the last two decades due to the more widespread availability of noninvasive diagnostic techniques and the publication of the first large cohort studies. CVT has a more varied clinical presentation than other stroke types and may be difficult to recognize, which may contribute to underdiagnosis. ${ }^{7}$ The clinical onset is more often subacute, but an acute presentation ( $<48$ hours) is also observed in about one-third of cases and a more chronic course is also possible, although it is less frequent. ${ }^{1}$ Symptoms and signs of CVT can be grouped in presenting syndromes, of which the most common are isolated intracranial hypertension syndrome, focal syndrome, and encephalopathy. ${ }^{7}$

The spectrum of parenchymal damage due to intracranial venous thrombosis includes a wide range of manifestations, from mild vasogenic edema to extensive parenchymal hemorrhage. Parenchymal brain lesions in CVT are often classified as nonhemorrhagic and hemorrhagic. ${ }^{8}$ These changes are better depicted in magnetic resonance imaging (MRI), which has increased sensitivity for the identification of parenchymal changes, especially the nonhemorrhagic, and also allows distinction between vasogenic and cytotoxic edema. In the ISCVT cohort, $64 \%$ of patients had parenchymal changes on computed tomography or MR. ${ }^{1}$ About half of the cases have a hemorrhagic component that often expands to the cortical surface, which may help in differentiating from the typical localization of parenchymal hypertensive bleeds. Juxtacortical flame-shaped lobar hemorrhages in the parasagittal frontal and parietal lobes, between the superficial

\section{received}

September 24, 2020

accepted after revision

December 7, 2020 (c) 2021. Thieme. All rights reserved. Georg Thieme Verlag KG,

Rüdigerstraße 14,

70469 Stuttgart, Germany
DOI https://doi.org/

10.1055/a-1332-3042. ISSN 0720-9355. 
and deep venous drainage systems, were described as a very specific finding in patients with CVT, particularly those with superior sagittal sinus thrombosis. ${ }^{9}$ The Brush sign, an MRI finding previously described in patients with ischemic stroke, can also be found in patients with CVT, in whom it is associated with ipsilateral parenchymal brain lesion, larger extent of thrombosis, and more severe clinical presentation. ${ }^{10}$ It corresponds to an abnormally accentuated hypointensity of the subependymal and medullary veins that can be seen in paramagnetic sensitive sequences ( $\mathrm{T} 2 *$ or susceptibility-weighted imaging) and is a likely marker of hypoperfusion and increased collateral circulation.

\section{Pathophysiology of Brain Damage}

Despite the progress made in the last years, significant evidence gaps persist in the understanding of the pathophysiology of brain damage and the mechanisms underlying the benefit of the available treatment strategies. Although there are several anecdotal reports of tissue recovery after recanalization, particularly following endovascular treatment, the association between early venous recanalization and tissue fate was not established until recently. In a prospective cohort study of patients treated with anticoagulation, we found that patients with persistent venous occlusion by day 8 after starting of anticoagulation treatment were more likely to have early worsening of nonhemorrhagic brain lesions. ${ }^{11}$ Importantly, patients achieving venous recanalization within the first week of therapeutic anticoagulation had a higher chance to show early regression of the nonhemorrhagic parenchymal lesions, comparing with patients with persistent occlusion. Besides suggesting that vessel recanalization is relevant for the evolution of brain damage, this finding raises the hypothesis that anticoagulation-induced early partial recanalization contributes to the clinical improvement often seen in these patients just a few days after treatment start. Contrary to what is described in ischemic stroke, lesions showing diffusion restriction were fully reversible in most cases, particularly in patients achieving early venous recanalization. ${ }^{11}$ This suggests that, in CVT, what was thought to represent cytotoxic edema can still represent viable brain tissue, and may imply that a longer time window for treatment options exists in CVT, even in patients showing the so-called venous infarction.

\section{Risk Factors}

The temporal profile of risk factors is a crucial feature because persistent predisposing conditions usually have specific implications on the long-term risk of recurrent venous thrombotic events. Therefore, risk factors are usually classified in permanent/nonmodifiable, such as genetic thrombophilia, and precipitant/transient, such as pregnancy or infection. ${ }^{12}$

CVT mostly affects young women, as the most common risk factors are female-specific, particularly pregnancy and hormonal contraception. Currently, oral contraceptives are the most frequent risk factor for CVT in women in Western countries. ${ }^{1}$ The overall risk of CVT associated with use of oral contraceptives was estimated to be seven times that of nonusers. ${ }^{13}$ However, the risk will be particularly high among those who carry other risk factors, such as obesity or hereditary thrombophilias. ${ }^{14,15}$ In fact, multiple risk factors are found in about half of patients. ${ }^{1}$ With regard to pregnancy and puerperium, the risk of any venous thrombosis was estimated to be increased by 4 - to 5 -fold during pregnancy and by 22 -fold during the first 6 weeks after delivery, compared with non-pregnant women. ${ }^{16,17}$ Like for other venous thrombotic events, the risk of pregnancyrelated CVT is higher in third trimester and puerperium, especially in the first 6 weeks postpartum, as confirmed by a recent case-control study conducted in high-income countries. ${ }^{18}$ Anemia and obesity have been also added to the list of risk factors for CVT over the past few years. ${ }^{15,19}$ Recently, there has been increased attention on the possible increased risk of CVT in patients with SARS-CoV-2 infection, as described for other thrombotic complications. ${ }^{20}$ Although the number of cases described so far is small ${ }^{21}$ underdiagnosis is likely, especially in patients with severe COVID-19. Further studies are warranted to confirm whether SARS-CoV-2 infection is a new risk factor for CVT.

With regard to the so-called permanent risk factors, the most common predisposing conditions are genetic prothrombotic disorders. ${ }^{1}$ Several publications from different regions of the world demonstrated that inherited thrombophilia increases the risk of CVT, especially in children. ${ }^{22}$ Acquired prothrombotic disorders are also found in a significant proportion of CVT cases, such as antiphospholipid syndrome (6$17 \%$ of cases ${ }^{12}$ ). Of note, there are several reports of CVT as a presenting manifestation of this syndrome. ${ }^{23}$ Cancer has been confirmed to be a powerful risk factor for the development of CVT, as the risk was estimated to be increased fivefold in patients with history of cancer. ${ }^{24}$ However, in patients with hematological neoplasms, the risk of CVT can be particularly increased, especially in the first year after diagnosis, with an estimated odds ratio for CVT of 86, according to a recent casecontrol study. ${ }^{23}$ Some specific forms of hematological cancer are more often associated with CVT, namely acute lymphoblastic leukemia ${ }^{25}$ and polycythemia vera. ${ }^{26}$ Specific hemotherapeutic agents, such as L-asparaginase, and other iatrogenic factors, such as lumbar punctures for intrathecal therapy, can also precipitate CVT. Several inflammatory conditions are also associated with increased risk of CVT. Behçet disease, is often complicated by CVT, with an estimated incidence of 3 cases per 1,000 person-years, ${ }^{27}$ corresponding to approximately 200 times the risk in the general population. In about $30 \%$ of these cases, CVT occurs in patients without a previous diagnosis of Behçet disease. ${ }^{27}$

Although the most common risk factors for CVT correspond to systemic disturbances, local conditions have also an established association with CVT, including head trauma, ${ }^{28}$ neurosurgical procedures, ${ }^{29}$ and infections, especially of the head and neck. ${ }^{30}$ Currently, these risk factors contribute to a smaller proportion of cases, despite considerable geographical variations in prevalence. Infectious causes, in particular, have substantially declined and septic CVT is uncommon nowadays, as a result of early treatment with antibiotics. Still, in 
contemporary multinational cohorts, infection was associated with 8 to $11 \%$ of cases of CVT. ${ }^{12}$ No underlying etiology or risk factor for CVT is found in approximately $15 \%$ of adult patients. ${ }^{1}$

\section{Venous Recanalization}

Considering data from previously published cohorts that included more than 800 patients treated with anticoagulation, a polled proportion of $85 \%$ of cases showed recanalization of at least one of the thrombosed vessels during followup (95\% confidence interval [CI], 80-89; $\left.I^{2}=58 \%\right) .{ }^{31}$ However, the methods and time points for evaluation varied across studies and there was significant heterogeneity in the results. ${ }^{31}$ To assess whether venous recanalization could occur earlier, even in patients treated only with anticoagulation, at what could be a more critical time window for the association with imaging and clinical outcomes, we have recently conducted a prospective study in which we were able to show that recanalization starts early in patients receiving therapeutic anticoagulation, as three quarters of patients had no persistent venous occlusion at day $8 .{ }^{11}$ However, venous recanalization progresses with time, and complete recanalization was only reached in about half of the patients at 90 days. Younger age was a predictor of early recanalization and there was a trend to an increased rate of early recanalization in patients showing the susceptibility vessel sign at admission. ${ }^{11}$ Although an association between persistent venous occlusion and worse functional outcome was found in the meta-analysis of previous cohort studies, ${ }^{31}$ that was not confirmed in this prospective cohort study with serial imaging at specific time points. ${ }^{11}$ Even so, the most severe presentations tended to be underrepresented and the sample size was likely to be unpowered to detect an association with long-term functional outcome, in a disease that most often has a favorable prognosis, as measured by the Rankin scale. Lack of complete recanalization was not a predictor of persistent headache. ${ }^{11}$

\section{Treatment}

The European guidelines on the management of CVT have been updated in $2017 .^{32}$ However, the quality of evidence was classified as low or very low for most of the recommendations, as they are mostly based on data from observational studies. Therapeutic anticoagulation is the standard treatment in CVT. The recommended antithrombotic treatment in the acute phase is heparin, either intravenous nonfractionated heparin or subcutaneous low-molecular-weight heparin (LMWH). Two trials, ${ }^{33,34}$ their meta-analysis, ${ }^{35}$ and one observational study ${ }^{36}$ showed a trend in favor of LMWH in mortality and functional outcomes. However, these studies had several methodological limitations, reducing the confidence on the conclusions.

Based on the hypothesis that rapid venous recanalization can prevent further deterioration and improve prognosis in severe CVT, use of endovascular treatment has been reported since the 1980s in hundreds of case reports and case series. The main endovascular approaches for the treatment of CVT are an intravascular chemical thrombolytic, mechanical thrombectomy, or the combined approach, using both techniques. A 2017 systematic review of case series with at least three cases treated with endovascular treatment included 235 patients. ${ }^{37}$ In this analysis, the authors found a mortality of $14 \%$ among patients receiving endovascular treatment, worsening or new intracranial hemorrhage in $9 \%$, and complete recovery in $35 \%$. Patients selected to the intervention were mostly severe cases, with $40 \%$ having encephalopathy or coma. The rate of complete recanalization was $69 \%$. A more recent systematic review that included 393 patients found that the combination of mechanical thrombectomy with local thrombolysis, when consistently performed across the cohort, tended to be associated with better outcomes. ${ }^{38}$ However, without a control group, no firm conclusions on the efficacy and safety of thrombectomy can be inferred from these analyses. Still, an evaluation of the United States Nationwide Inpatient Sample between 2004 and 2014 estimated that approximately $3 \%$ of all CVT patients received endovascular treatment (1,248 patients). ${ }^{39}$ There was high heterogeneity in the used techniques and no information was available on the recanalization rate. Due to the very low quality of available evidence, the European guidelines do not make any recommendation, and suggest not using endovascular treatment in acute CVT patients with a pretreatment low risk of poor outcome. ${ }^{32}$ Recently, the neutral results of the first randomized comparison of adjunctive EVT versus standard treatment with anticoagulation have further increased the uncertainty on the effectiveness of this intervention in patients with severe CVT. ${ }^{40}$ The TO-ACT trial included patients having at least a predictor of poor outcome and decisions regarding the protocol for the endovascular intervention were left to the treating interventionalist. The trial was terminated prematurely for futility, after inclusion of 67 patients, and no difference in clinical outcome was detected between those allocated to anticoagulation only or adjunctive endovascular treatment (modified Rankin's scale of $0-1$ in $67 \%$ vs. 68\% patients, in the intervention and standard treatment arm, respectively). Perforation of sinus or cortical vein was reported in 3 three cases (9\%), although this was not associated with symptomatic bleeding. ${ }^{40}$ Further research on the pathophysiology of brain lesions, prognostic markers, and intervention techniques directed to the venous system should contribute to improve patient selection, as well as the safety and efficacy of these neuro vascular interventions in CVT.

Herniation due to unilateral mass effect is the major cause of death in CVT. ${ }^{41}$ Although the evidence for the use of decompressive surgery in CVT comes from uncontrolled studies, it consistently suggests patients with impending brain herniation are best treated with decompressive surgery. Particularly, several single-center series and one multicenter registry and systematic review of individual patients showed that decompressive surgery is lifesaving and often results in good functional outcome. ${ }^{42-44}$ A prospective register of decompressive surgery in CVT (DECOMPRESS-2) that included more than 100 patients has been recently finished and results are expected soon.

Treatment of the associated conditions/risk factors and management of symptoms and complications is also an 
important part of the management of patients with CVT. Acute symptomatic seizures are a common manifestation, occurring in about one-third of patients. ${ }^{45}$ Late seizures occur in $11 \%$ of patients, especially in patients with intracranial bleeding, acute symptomatic seizures, or who underwent decompressive hemicraniectomy. ${ }^{46}$

\section{Long-Term Management}

After the initial period of parenteral anticoagulation, oral anticoagulants should be started, unless there is a specific contraindication. Recently, an exploratory randomized controlled trial (RE-SPECT CVT) comparing the efficacy and safety of dabigatran $150 \mathrm{mg}$, bid, versus dose-adjusted warfarin (INR 2-3) during 6 months, was completed, after allocating $60 \mathrm{CVT}$ patients to each treatment arm. ${ }^{47}$ Oral anticoagulation was started 5 to 15 days after the beginning of parenteral anticoagulation, in clinically stable patients. The risk of recurrent venous thrombotic events was low in both arms, with similar rates of bleeding, suggesting that both dabigatran and warfarin may be safe and effective for preventing recurrent thrombotic events in patients with CVT. There is an ongoing open-label phase II trial assessing the safety of rivaroxaban compared with heparin with subsequent transition to warfarin or continued $\mathrm{LMWH}$, in patients with CVT (SECRET trial NCT03178864). Contrary to the previous trial, oral anticoagulation can be started in the acute phase, instead of parenteral anticoagulation. The EINSTEIN Junior study is also evaluating the efficacy and safety of rivaroxaban in children with venous thrombosis, including CVT (NCT02234843).

Based on the largest cohorts studies with long-term follow-up, the risk of recurrent events in patients with previous CVT is estimated to be around 2 events per 100 patient-years for recurrent CVT and approximately 4 events per 100 patient-years for noncerebral venous thrombosis, ${ }^{2,48}$ with most events occurring after anticoagulant therapy withdrawal. Importantly, the rate of recurrence seems to persist with time, as demonstrated in a long-term French cohort study including 187 patients, in which $18 \%$ of patients had a recurrent thrombotic event in the 10 years that followed CVT. ${ }^{49}$ In adults, risk factors for recurrence of CVT are male gender, myeloproliferative diseases (polycythemia vera or essential thrombocythemia), prior venous thrombotic events, and severe thrombophilia. ${ }^{50-52}$

Oral anticoagulation after the acute phase of CVT contributes to the prevention of further venous thrombotic events, including recurrence of CVT. However, there have been no randomized controlled trials or prospective controlled studies assessing optimal duration of oral anticoagulation in this setting. ${ }^{32}$ The first trial that addresses this question (EXtending Oral Anticoagulant treatment after acute Cerebral Vein Thrombosis, EXCOA-CVT) is currently ongoing. ${ }^{53}$ Meanwhile, oral anticoagulation is usually maintained for 3 to 12 months after acute CVT. ${ }^{32}$ In patients with genetic or acquired prothrombotic conditions (e.g., antithrombin deficiency, antiphospholipid syndrome, and active cancer) at high risk of recurrent venous thrombosis, or with history of prior venous thrombotic events, lifelong anticoagulation should be considered. ${ }^{30}$

Future health conditions associated with increased risk of recurrent thrombotic events should also be a concern. As CVT is more common in women at fertile age and thromboembolism is a leading cause of maternal mortality, safety of future pregnancies is a common question in clinical practice in patients with history of CVT. Specifically, besides counseling, a decision has to be made on whether to offer antithrombotic prophylaxis or not during pregnancy and/or puerperium. The currently available evidence, which was substantially increased in the last years by new follow-up studies $^{54}$ and systematic reviews, ${ }^{5,56}$ supports medical counseling in favor of not contraindicating future pregnancy in women with previous CVT, although they should be informed on the increased risks. ${ }^{32}$ Also, in women with a history of CVT, heparin prophylaxis was associated with lower rates of pregnancy-related recurrent thrombotic events and a significantly lower risk of miscarriage. ${ }^{54-56}$

\section{Prognosis}

Overall, death or dependence occurs in approximately $15 \%$ of patients, despite medical treatment. ${ }^{1}$ However, mortality rates above $30 \%$ have been reported in case series of patients with severe CVT admitted to an intensive care unit, ${ }^{57,58}$ mostly due to large herniating brain lesions.

The clinical course of CVT is unpredictable in the first days after diagnosis, and about one quarter of patients deteriorate in the acute phase. ${ }^{1}$ Among the surviving patients, approximately 6 to $10 \%$ have severe and permanent disability. ${ }^{1}$ Predictors of poor outcome include older age, male sex, coma, mental status disorder, intracerebral hemorrhage, thrombosis of the deep venous system, infection of the central nervous system, cancer, and hyperglycemia at admission. ${ }^{1,59}$

In recent years, there was increasing interest in the subtle consequences of CVT in patients with apparently full recovery. Even though approximately $80 \%$ of patients recover from CVT without physical disability, many of these patients experience residual chronic symptoms. About half of patients report headache during follow-up, and severe headaches that require bed rest or hospital admission persist in $14 \%$ of patients. ${ }^{1}$ More than half of survivors of CVT report subtle neuropsychological difficulties or depression. These complaints are often associated with a negative effect on employment status, and 20 to $40 \%$ of patients are unable to return to their prior working life. ${ }^{60}$

\section{Conflict of Interest}

Dr. Aguiar de Sousa reports nonfinancial support from Boehringer Ingelheim, outside the submitted work.

\section{References}

1 Ferro JM, Canhão P, Stam J, Bousser MG, Barinagarrementeria FISCVT Investigators. Prognosis of cerebral vein and dural sinus thrombosis: results of the International Study on Cerebral Vein and Dural Sinus Thrombosis (ISCVT). Stroke 2004;35(03): $664-670$ 
2 Dentali F, Poli D, Scoditti U, et al;CErebral VEin Thrombosis International Study Investigators. Long-term outcomes of patients with cerebral vein thrombosis: a multicenter study. J Thromb Haemost 2012;10(07):1297-1302

3 Ferro JM, Canhão P, Bousser MG, Stam J, Barinagarrementeria FISCVT Investigators. Cerebral vein and dural sinus thrombosis in elderly patients. Stroke 2005;36(09):1927-1932

4 Zuurbier SM, Hiltunen S, Lindgren E, et al. Cerebral venous thrombosis in older patients. Stroke 2018;49(01):197-200

5 Coutinho JM, Zuurbier SM, Aramideh M, Stam J. The incidence of cerebral venous thrombosis: a cross-sectional study. Stroke 2012; 43(12):3375-3377

6 Otite FO, Patel S, Sharma R, et al. Trends in incidence and epidemiologic characteristics of cerebral venous thrombosis in the United States. Neurology 2020;95(16):e2200-e2213

7 Ferro JM, Canhão P, Aguiar de Sousa D. Cerebral venous thrombosis. Presse Med 2016;45(12, Pt 2):e429-e450

8 Ferro JM, Canhão P, Bousser M-G, Stam J, Barinagarrementeria F, Stolz EISCVT Investigators. Cerebral venous thrombosis with nonhemorrhagic lesions: clinical correlates and prognosis. Cerebrovasc Dis 2010;29(05):440-445

9 Coutinho JM, van den Berg R, Zuurbier SM, et al. Small juxtacortical hemorrhages in cerebral venous thrombosis. Ann Neurol 2014;75(06):908-916

10 Aguiar de Sousa D, Lucas Neto L, Jung S, et al. Brush sign is associated with increased severity in cerebral venous thrombosis. Stroke 2019;50(06):1574-1577

11 Aguiar de Sousa D, Lucas Neto L, Arauz A, et al. Early recanalization in patients with cerebral venous thrombosis treated with anticoagulation. Stroke 2020;51(04):1174-1181

12 Silvis SM, de Sousa DA, Ferro JM, Coutinho JM. Cerebral venous thrombosis. Nat Rev Neurol 2017;13(09):555-565

13 Amoozegar F, Ronksley PE, Sauve R, Menon BK. Hormonal contraceptives and cerebral venous thrombosis risk: a systematic review and meta-analysis. Front Neurol 2015;6:7

14 Martinelli I, Sacchi E, Landi G, Taioli E, Duca F, Mannucci PM. High risk of cerebral-vein thrombosis in carriers of a prothrombin-gene mutation and in users of oral contraceptives. N Engl J Med 1998; 338(25):1793-1797

15 Zuurbier SM, Arnold M, Middeldorp S, et al. Risk of cerebral venous thrombosis in obese women. JAMA Neurol 2016;73(05): 579-584

16 Pomp ER, Lenselink AM, Rosendaal FR, Doggen CJM. Pregnancy, the postpartum period and prothrombotic defects: risk of venous thrombosis in the MEGA study. J Thromb Haemost 2008;6(04): 632-637

17 Sultan AA, West J, Tata LJ, Fleming KM, Nelson-Piercy C, Grainge MJ. Risk of first venous thromboembolism in and around pregnancy: a population-based cohort study. Br J Haematol 2012;156 (03):366-373

18 Ferro JM, de Sousa DA, Oliveira S. Cerebral venous thrombosis: genetic aspects. In: Stroke Genetics. 2nd ed. Springer Link; 2017: 295-326

19 Coutinho JM, Zuurbier SM, Gaartman AE, et al. Association between anemia and cerebral venous thrombosis: case-control study. Stroke 2015;46(10):2735-2740

20 Cavalcanti DD, Raz E, Shapiro M, et al. Cerebral venous thrombosis associated with COVID-19. AJNR Am J Neuroradiol 2020;41(08): 1370-1376

21 Baldini T, Asioli GM, Romoli M, et al. Cerebral venous thrombosis and SARS-CoV-2 infection: a systematic review and meta-analysis. Eur J Neurol 2021. doi: 10.1111/ene.14727

22 Marjot T, Yadav S, Hasan N, Bentley P, Sharma P. Genes associated with adult cerebral venous thrombosis. Stroke 2011;42(04): 913-918

23 Shlebak A. Antiphospholipid syndrome presenting as cerebral venous sinus thrombosis: a case series and a review. J Clin Pathol 2016;69(04):337-343
24 Silvis SM, Hiltunen S, Lindgren E, et al. Cancer and risk of cerebral venous thrombosis: a case-control study. J Thromb Haemost 2018;16(01):90-95

25 Zuurbier SM, Lauw MN, Coutinho JM, et al. Clinical course of cerebral venous thrombosis in adult acute lymphoblastic leukemia. J Stroke Cerebrovasc Dis 2015;24(07):1679-1684

26 Passamonti SM, Biguzzi E, Cazzola M, et al. The JAK2 V617F mutation in patients with cerebral venous thrombosis. J Thromb Haemost 2012;10(06):998-1003

27 Aguiar de Sousa D, Mestre T, Ferro JM. Cerebral venous thrombosis in Behçet's disease: a systematic review. J Neurol 2011;258 (05):719-727

28 Netteland DF, Mejæænder-Evjensvold M, Skaga NO, Sandset EC, Aarhus M, Helseth E. Cerebral venous thrombosis in traumatic brain injury: a cause of secondary insults and added mortality. J Neurosurg 2020; (epub ahead of print). Doi: 10.3171/2020.4. JNS20511

29 Benjamin CG, Sen RD, Golfinos JG, et al. Postoperative cerebral venous sinus thrombosis in the setting of surgery adjacent to the major dural venous sinuses. J Neurosurg 2018;(October):1-7

30 Zuurbier SM, Coutinho JM, Stam J, et al;ISCVT Investigators. Clinical outcome of anticoagulant treatment in head or neck infection-associated cerebral venous thrombosis. Stroke 2016; 47(05):1271-1277

31 Aguiar de Sousa D, Lucas Neto L, Canhão P, Ferro JM. Recanalization in cerebral venous thrombosis. Stroke 2018;49(08): $1828-1835$

32 Ferro JM, Bousser MG, Canhão P, et al;European Stroke Organization. European Stroke Organization guideline for the diagnosis and treatment of cerebral venous thrombosis - endorsed by the European Academy of Neurology. Eur J Neurol 2017;24(10): 1203-1213

33 Misra UK, Kalita J, Chandra S, Kumar B, Bansal V. Low molecular weight heparin versus unfractionated heparin in cerebral venous sinus thrombosis: a randomized controlled trial. Eur J Neurol 2012;19(07):1030-1036

34 Afshari D, Moradian N, Nasiri F, Razazian N, Bostani A, Sariaslani P. The efficacy and safety of low-molecular-weight heparin and unfractionated heparin in the treatment of cerebral venous sinus thrombosis. Neurosciences (Riyadh) 2015;20(04):357-361

35 Qureshi A, Perera A. Low molecular weight heparin versus unfractionated heparin in the management of cerebral venous thrombosis: a systematic review and meta-analysis. Ann Med Surg (Lond) 2017;17:22-26

36 Coutinho JM, Ferro JM, Canhão P, Barinagarrementeria F, Bousser MG, Stam JISCVT Investigators. Unfractionated or low-molecular weight heparin for the treatment of cerebral venous thrombosis. Stroke 2010;41(11):2575-2580

37 Ilyas A, Chen CJ, Raper DM, et al. Endovascular mechanical thrombectomy for cerebral venous sinus thrombosis: a systematic review. J Neurointerv Surg 2017;9(11):1086-1092

38 Lewis W, Saber H, Sadeghi M, Rajah G, Narayanan S. Transvenous endovascular recanalization for cerebral venous thrombosis: a systematic review and meta-analysis. World Neurosurg 2019; 130:341-350

39 Siddiqui FM, Weber MW, Dandapat S, et al. Endovascular thrombolysis or thrombectomy for cerebral venous thrombosis: study of nationwide inpatient sample 2004-2014. J Stroke Cerebrovasc Dis 2019;28(06):1440-1447

40 Coutinho JM, Zuurbier SM, Bousser MG, et al;TO-ACT Investigators. Effect of Endovascular treatment with medical management vs standard care on severe cerebral venous thrombosis: the TOACT randomized clinical trial.JAMA Neurol 2020;77(08):966-973

41 Canhão P, Ferro JM, Lindgren AG, Bousser MG, Stam J, Barinagarrementeria FISCVT Investigators. Causes and predictors of death in cerebral venous thrombosis. Stroke 2005;36(08):1720-1725

42 Ferro JM, Crassard I, Coutinho JM, et al;Second International Study on Cerebral Vein and Dural Sinus Thrombosis (ISCVT 2) 
Investigators. Decompressive surgery in cerebrovenous thrombosis: a multicenter registry and a systematic review of individual patient data. Stroke 2011;42(10):2825-2831

43 Zuurbier SM, Coutinho JM, Majoie CBLM, Coert BA, van den Munckhof P, Stam J. Decompressive hemicraniectomy in severe cerebral venous thrombosis: a prospective case series. J Neurol 2012;259(06):1099-1105

44 Raza E, Shamim MS, Wadiwala MF, Ahmed B, Kamal AK. Decompressive surgery for malignant cerebral venous sinus thrombosis: a retrospective case series from Pakistan and comparative literature review. J Stroke Cerebrovasc Dis 2014;23(01):e13-e22

45 Lindgren E, Silvis SM, Hiltunen S, et al. Acute symptomatic seizures in cerebral venous thrombosis. Neurology 2020;95 (12):e1706-e1715

46 Sánchez van Kammen M, Lindgren E, Silvis SM, et al. Late seizures in cerebral venous thrombosis. Neurology 2020;95(12): e1716-e1723

47 Ferro JM, Coutinho JM, Dentali F, et al;RE-SPECT CVT Study Group. Safety and efficacy of dabigatran etexilate vs doseadjusted warfarin in patients with cerebral venous thrombosis: a randomized clinical trial. JAMA Neurol 2019;76(12): $1457-1465$

48 Miranda B, Ferro JM, Canhão P, et al;ISCVT Investigators. Venous thromboembolic events after cerebral vein thrombosis. Stroke 2010;41(09):1901-1906

49 Palazzo P, Agius P, Ingrand P, et al. Venous thrombotic recurrence after cerebral venous thrombosis: a long-term follow-up study. Stroke 2017;48(02):321-326

50 Martinelli I, Bucciarelli P, Passamonti SM, Battaglioli T, Previtali E, Mannucci PM. Long-term evaluation of the risk of recurrence after cerebral sinus-venous thrombosis. Circulation 2010;121(25): 2740-2746

51 Lim HY, Ng C, Donnan G, Nandurkar H, Ho P. Ten years of cerebral venous thrombosis: male gender and myeloproliferative neo- plasm is associated with thrombotic recurrence in unprovoked events. J Thromb Thrombolysis 2016;42(03):423-431

52 Pires GS, Ribeiro DD, Oliveira JAQ et al. Risk factors associated with recurrent venous thromboembolism after a first cerebral venous thrombosis event: a cohort study. Thromb Res 2019; 178:85-90

53 Miranda B, Aaron S, Arauz A, et al. The benefit of EXtending oral antiCOAgulation treatment (EXCOA) after acute cerebral vein thrombosis (CVT): EXCOA-CVT cluster randomized trial protocol. Int J Stroke 2018;13(07):771-774

54 Aguiar de Sousa D, Canhão P, Crassard I, et al;ISCVT-2-PREGNANCY Investigators. Safety of pregnancy after cerebral venous thrombosis results of the ISCVT (international study on cerebral vein and dural sinus thrombosis)-2 PREGNANCY study. Stroke 2017;48(11):3130-3133

55 Aguiar de Sousa D, Canhão P, Ferro JM. Safety of pregnancy after cerebral venous thrombosis: a systematic review. Stroke 2016;47 (03):713-718

56 Aguiar de Sousa D, Canhão P, Ferro JM. Safety of pregnancy after cerebral venous thrombosis: systematic review update. J Neurol 2018;265(01):211-212

57 Kowoll CM, Kaminski J, Weiß V, et al. Severe cerebral venous and sinus thrombosis: clinical course, imaging correlates, and prognosis. Neurocrit Care 2016;25(03):392-399

58 Soyer B, Rusca M, Lukaszewicz AC, et al. Outcome of a cohort of severe cerebral venous thrombosis in intensive care. Ann Intensive Care 2016;6(01):29

59 Zuurbier SM, Hiltunen S, Tatlisumak T, et al. Admission hyperglycemia and clinical outcome in cerebral venous thrombosis. Stroke 2016;47(02):390-396

60 Hiltunen S, Putaala J, Haapaniemi E, Tatlisumak T. Long-term outcome after cerebral venous thrombosis: analysis of functional and vocational outcome, residual symptoms, and adverse events in 161 patients. J Neurol 2016;263(03):477-484 